\title{
THE KEY OF BULK WAREHOUSE GRAIN QUANTITY RECOGNITION Rectangular Benchmark Image Recognition
}

\author{
Ying $\operatorname{Lin}^{1,2, *}$, Yang $\mathrm{Fu}^{1}$ \\ ${ }^{1}$ College of management, Chong Qing Jiao Tong University, Chongqing, China, 400074 \\ ${ }^{2}$ School of Electronic Information Engineering, Tian Jin University, Tianjin, China, 300072 \\ * Corresponding author, Address: No. 66 Xuefudadao, Nanan district, Chongqing Municipality, \\ 400074, P. R. China, Tel: +86-23-66876662, Email: Linyingdyh@yahoo.com.cn
}

\begin{abstract}
According to requests of bulk warehouse grain quantity recognition, we take the scene video as identified object to obtain the object's boundary from the result of edge detection difference iterative analysis. By using region iterative threshold value of gradient operator fitted closely with identified target carries to execute the picture characteristic second-extract and then to carrying on rectangular benchmark judgment using the membership functions of fuzzy recognition, we adopt the Visual $\mathrm{C}++$ realized this recognition algorithm. And the experimental results show that this recognition algorithm effectively enhances the anti-jamming, robustness and the recognition precision and effect.
\end{abstract}

Keywords: edge detection difference, fuzzy recognition, membership functions, iterative analysis

\section{INTRODUCTION}

From the last 20 years' practice of storage grain regulatory in China, the quantity of grain reserves supervision and auditing is still manual regulation. Because of the geographical dispersion of reserve granary and the features of whole process and real-time supervising, it leads to the failure of grain reserves effective supervision. So it is necessary to adopt a computerized intelligent recognition technology to achieve the precious quantity of grain reserves automatic monitoring and auditing in a smart method. The key for video-based grain reserves automatic monitoring and auditing technology is 
based on the result of scenes video image recognition to calculate the quantity of grain granary.

From (Fig. 1), it indicates that the bulk of the reserve granary bottom of the area set $\mathrm{S}$, grain density of $\rho$ reserve granary high set $\mathrm{T}$ are known factors, grain surface above the benchmark length is L, M is the quality of grain, then:

Grain quality $\mathrm{M}=\mathrm{V} \times \rho$

Where $\mathrm{V}=\mathrm{S} \times \mathrm{H}, \quad \mathrm{H}=\mathrm{T}-\mathrm{L}$

According to the above statements, the key of grain quantity recognition is the identification and estimation of reserve granary benchmark. The key problem of real-scene recognition of granary reserves benchmark is how to identify the object boundary and discriminate the identified targets. The existing Robert operator partial detection methods can effectively extract the object boundary (M.D. Kelly, 1973). But it has poor adaptive ability for the interference, and also the algorithm efficiency is not high efficiency enough. Therefore, seeking stronger anti-jamming detection methods and target's characteristic judgment method has become an issue that must be addressed. In this paper we introduce a combining intelligent detection method to identify the benchmark by Robert Operator local detection and the overall threshold detection algorithm (Canny J., 1986).

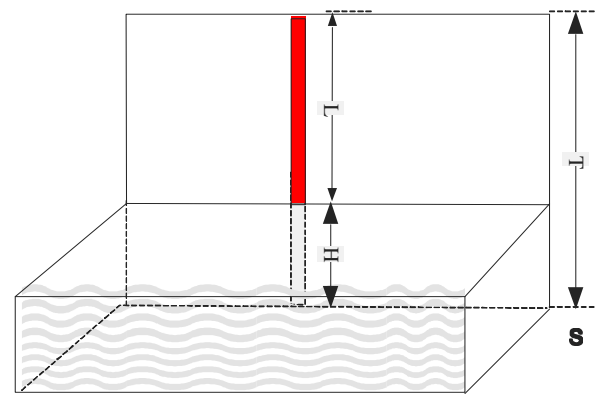

Fig.1. Sketch map of bulk grain warehouse

\section{INTELLIGENT DETECTION METHOD}

The use of computer recognition is mainly by computer automatic identification and understanding of images. To this purpose, the decomposition of image that contains a large variety of characteristic information as a key step. The accuracy level of intelligent recognition is directly related with the shape, color and specific size of identified targets. A key technology of image detection is how to determine ROI (region of interests), the image divided by Robert operator partial detection operator gradient method and operator of regional iteration threshold image segmentation algorithm combination of images to the second feature 
extraction, a new intelligent detection method is involved (Pursuing technology, 2006).

This method includes following three major steps.

1. Color extraction: The setting of real-scene should fully consider the issue of the benchmark's color. Firstly image is converted to color space, extracting specific benchmark for the red, tentatively identified ROI region.

2. Edge extraction: Based on the edge of extraction from the red border regions, we use Robert segmentation local edge detection operator and combine with the iterative threshold to extract the pixel of the same difference, to some extent reduce the computational time and the complexity of the recognition (Cheng Xiao-chun, 2006).

3. Rectangular benchmark judgment: Judging from the extracted targets, through certain principles found matching rectangular regional area.

\subsection{Color extraction}

Based on the specific color of rectangular benchmark, firstly we extract the color from the hue, saturation and intensity model (HSI) color space, initially identifying ROI region. In the actual image acquisition process, from the acquisition of Monitor RGB format for images, since the first need to achieve a conversion to RGB space HSI space (Kim, V. \& L. Yaroslavskii, 1986).

First step will be a normalized mode:

$$
r=\frac{R}{255}, b=\frac{B}{255}, g=\frac{G}{255}
$$

Then proceed into:

$$
H=90^{\circ}-\arctan \left(\frac{F}{\sqrt{3}}\right) \times \frac{180^{\circ}}{\pi}+\{0, g>b ; 180, g<b\}
$$

Where:

$$
F=\frac{2 r-g-b}{g-b}
$$

It is known from HIS color model picture that one can extract the regions of arbitrary color accord to our needs (Tou, J.T. \& R.C., 1981). In the benchmark identification process, the design needs extract the red region, the corresponding distribution of red region is $315^{\circ}-360^{\circ}$ and $0^{\circ}-23^{\circ}$, we can determine the initial ROI region.

\subsection{Edge extraction}

The main purpose of edge extraction is more accurately to reduce the number of pixels in ROI region, on the basis of color extraction result 
acquired, in order to effectively improve the accuracy of feature extraction. Edge extraction carried on the source gray image, using the rate of change of intensity and direction of changes in the method Robert boundary segmentation local edge detection operator method checks each pixel point for the neighborhood, and the completion of the pixels in a neighborhood of gray rate of changes, which in the direction of quantifying the identification, then to the border Robert segmentation local edge detection operator method calculate the edge pixels gradient operator basis second operational threshold will have the same rate of difference of gray pixels constitute closure and connectivity region. (Marr, D. \& Hildreth, E., 1980)

Then, For two-dimensional image $x, y$ respectively, on behalf of the pixels in a two-dimensional pixel-point benchmark of the abscissa, longitudinal coordinates, the position $f(x, y)$ of the gradient can be expressed as a vector, using $G_{x}$ and $G_{y}$ Specific formula as follows: Gradient vector can be expressed as the following:

$$
\nabla f(x, y)=\left[\begin{array}{l}
G_{x} \\
G_{y}
\end{array}\right]=\left(\begin{array}{l}
\frac{\partial f(x, y)}{\partial x} \\
\frac{\partial f(x, y)}{\partial y}
\end{array}\right)
$$

Set $\theta_{\mathrm{r}}$ Represent gradient direction:

$$
\theta_{\mathrm{r}}=\tan ^{-1}\left(f_{\mathrm{x}} / f_{\mathrm{y}}\right)
$$

In the direction of $\theta_{\mathrm{r}}$ the rate of change velocity:

$$
g(x, y)=|\nabla f(x, y)|=\sqrt{\left(\frac{\partial f(x, y)}{\partial x}\right)^{2}+\left(\frac{\partial f(x, y)}{\partial y}\right)^{2}}
$$

In the margin calculation gradient operator is equivalent to the following two ways calculated norm:

$$
g(x, y)=\left|\frac{\partial f(x, y)}{\partial x}\right|+\left|\frac{\partial f(x, y)}{\partial y}\right|
$$

Or to use infinity norm:

$$
g(x, y)=\max \left(\left|\frac{\partial f(x, y)}{\partial x}\right|,\left|\frac{\partial f(x, y)}{\partial y}\right|\right)
$$

In fact, the use of computers to handle most of the images is targeted at digital images, in the field of digital image processing, using the above difference for alternative differential. The definition of the form is as follows: 


$$
\begin{aligned}
& f_{x}(x, y)=f(x, y)-f(x-1, y) \\
& f_{y}(x, y)=f(x, y)-f(x, y-1) \\
& g(x, y)=f_{x}(x, y)+f_{y}(x, y)
\end{aligned}
$$

The visualization of image threshold processing makes it easy to realize the image segmentation application; this is also the main factor to choose the method of threshold processing (Pavlidis, T., 1982). Adopted the idea of threshold to (i.e. difference value) implement threshold segmentation on the gradient operator, the key issue of the threshold treatment is how to choose appropriate threshold segmentation. Difference is the threshold for the acquisition to take recursive iterative approach is relative to the test selection of simple threshold segmentation (i.e. the average of the overall difference), the accuracy of operational results is greatly improved (J. Kittler, M. Hatef, R.P.W. Duin, and J. Matas, 1998). Specifically operational steps as (Fig. 2) shows:

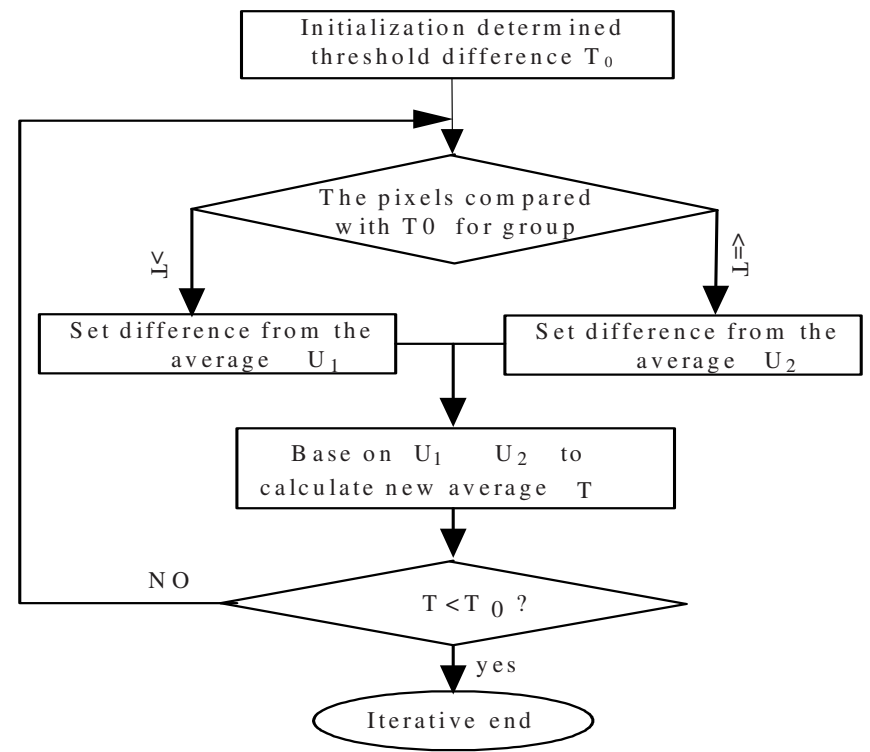

Fig. 2. Process of image division disposal

1. The process of edge calculates the gradient operator's expectations as the initial sub-threshold;

$$
E(z)=T_{0}=\frac{\sum_{i=1}^{n} g(x, y)}{n}
$$

2. Using $\mathrm{T}_{0}$ for image segmentation, all pixels divided into two groups: $\mathrm{V}_{1}$ group: $g(x, y)>\mathrm{T}_{0}$ pixels composition; $\mathrm{V}_{2}$ group: $g(x, y)<=\mathrm{T}_{0}$ pixels composition; 
3. The calculation of two different sets of internal difference, on average, and recorded as $\mu_{1}$ and $\mu_{2}$;

4. And the value of difference for the average operation, to calculate the difference the new threshold: $\mathrm{T}_{0}=1 / 2\left(\mu_{1}+\mu_{2}\right)$;

5. Repeating the Step 2 to Step 4 of the calculation process, until successive iteration is less than the value of $\mathrm{T}_{0}$ prior estimate the parameters $\mathrm{T}_{0}$ stop iteration. (Gao, J., Zhou, M. \& Wang, H., 2001)

\subsection{Rectangular benchmark judgment}

During the above process, there is likely to be the noise induced by the hardware or in the transmission process generated by the channel, because of the background and there are some isolated the noisy points (J.F. Canny, 1986), in order to improve recognition accuracy in the design, base on the above handled regional ROI pixels. But Robert conducting boundary segmentation local edge detection operator gradient method and operate of regional iteration threshold Image cutting algorithm, combining regional approach to ROI for the second marginal results may still be some noisy points misjudgment as the ROI edge pixels (T. Poggio, H. Voorhees and A. Yuille, 1985).

Firstly, reducing the noise of extracted gray image, according to the practical application of the design need to take connectivity judgment methods to judge, given the size of the noise, the design of the introduction of $2 * 2$ connectivity matrix judgment. Using $320 * 240$ to the scene graph as template, and a description of the specific approach is shown in (Fig. 3).

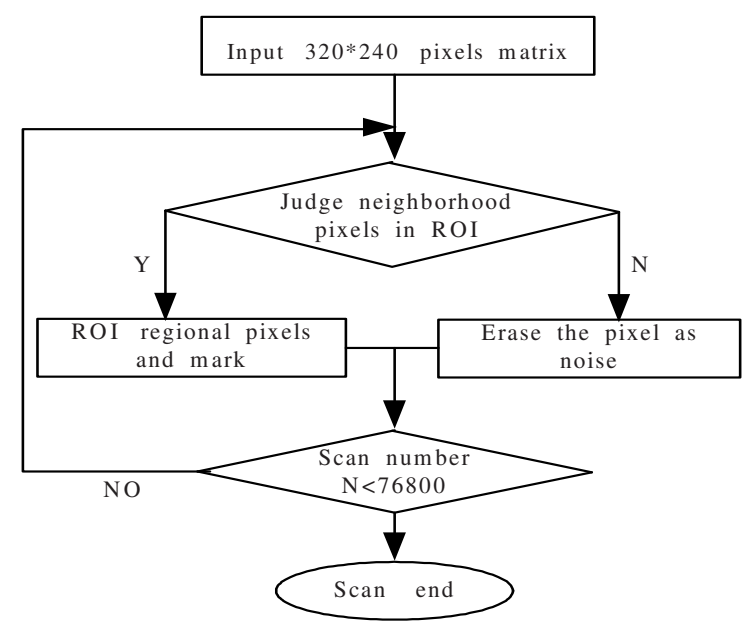

Fig. 3. Remove of image noise disposal

Secondly, conducting the pattern recognition based on the ROI edge region has been labeled, preparing for the conduct of the length calculations. Geometry of the pattern recognition is the key to determine the geometry 
and its membership function (i.e. fuzzy degrees), the right has been labeled rectangular gray two marginal value of the map edge smoothing and the corresponding pixel matrix conversion (Duda, R.O. \& Hart, P.E., 1973).

A, B, C, D quadrilateral as four interior angle, a, b represent two adjacent edges Quadrilateral length, this is the number of pixels, and fuzzy correlation coefficient, $\rho_{1}, \rho_{2}$ are respective the relative factor.

Angle membership functions:

$$
(R E)\left(u_{1}\right)=1-\rho_{1} \frac{1}{90}\left[\left(A-90^{\circ}\right)+\left(B-90^{\circ}\right)+\left(C-90^{\circ}\right)+\left(D-90^{\circ}\right)\right]
$$

Adjacent edges membership function:

$$
(R E)\left(u_{2}\right)=1-\rho_{2} \frac{a}{b}
$$

When had withdrawn the rectangular ROI regional membership meets the following conditions:

$$
(R E)\left(u_{1}\right)>=0.8 \text { and }(R E)\left(u_{2}\right)>=0.8
$$

Then the computer will determine the ROI for the region to be identified rectangular benchmark.

\section{THE EXPERIMENTAL ANALYSIS AND CONCLUSIONS}

From the above implement, we can educe the conclusion of this intelligent recognition, as follows:

(1) Divided by the border Robert partial edge detection operator iterative methods to extract rectangular benchmark is feasible (Fu, K.S. \& Mui, J.K., 1981). Advanced intelligent recognition technology has certain value in practical application of the regulatory process of the grain reserves of realtime monitoring.

(2) The methodology used HIS color space conversion, the use of H-hue color space, so it has good robustness under different light circumstance.

(3) Using the design methods in the actual recognition, all of the 10 samples of the rectangular benchmark can be correctly recognized, and there is not misjudgment, that is the correct detection rate is $100 \%$.

Based on the grain reserves automatic video monitoring and auditing, the key technology is through the recognition of real-scenes video image to acquire the quantity of grain storage. In this paper, a boundary segmentation Robert partial edge detection operator methods and iterative threshold regional gradient operator segmentation algorithm are combined to complete the edge of the ROI region extraction, and use a fuzzy membership function of recognition for rectangular benchmark judgment, the scheme is simple 
and the calculation is not too much, effectively remedy the processing failures that aroused by the edge detection operators to noisy image, and have good effect of edge detection. But the result of some processed image still has a certain edge of the width. For the department of grain management require a high precision for grain quantity calculation, the width of the benchmark length will result in errors for the subsequent calculation of grain quantity, therefore, the further refinement of the image edge extraction still needs further study and discussion.

\section{ACKNOWLEDGEMENTS}

This work is supported under the Bulk Warehouse Grain Quantity Recognition, a project (2007) funded by the Finance Bureau of Chongqing Municipality, P. R. China.

\section{REFERENCES}

Canny, J. (1986). A Computational Approach to Edge Detection [J], IEEE Transations Pattern Analytical Machine Intelligent 8: 679-698.

Cheng Xiao-chun (2006). A method of shape recognition [J], Pattern Recognition and Artificial Intelligence 6: 126-132.

Duda, R.O. \& Hart, P.E. (1973). Pattern Classification and Scene Analysis [J], New York: Wiley.

Fu, K. S. \& Mui, J. K. (1981). A Survey of Image Segmentation. Pattern Recognition [J], IEEE Transactions on Pattern Analysis and Machine Intelligence 13(1): 3-16.

Gao, J., Zhou, M. \& Wang, H. (2001). A Threshold and Region Growing Combined Method for Filament Disappearance Area Detection in Solar Images [J], In Proceedings of The Conference on Information Sciences and Systems. The John Hopkins University.

Huertas, A. \& Medioni G. (1986). Detection of Intensity Changes with Sub-Pixel Accuracy using Laplacian-Gaussian Masks [J], IEEE Transactions Pattern Recognition Machine Intelligent 8(5): 651-664.

J.F. Canny (Nov 1986), A Computational Approach to Edge Detection [J], IEEE PAMI, Vol. 8, No. 6.

J. Kittler, M. Hatef, R.P.W. Duin, and J. Matas. (1998). On combining classifiers [J]. IEEE Transactions on Pattern Analysis and Machine Intelligence 20(3): 226-239.

Kim, V. \& L. Yaroslavskii, (1986). Rank algorithms for picture processing [J], Comput Vision, Graphics, and Image Process 35: 234-258.

M.D. Kelly, Edge Detection in Pictures by Computer Planning [J], Machine Intelligence,Vol. 6 (American Elsevier, New York, 1973), pp. 397-409.

Marr, D. \& Hildreth, E. (1980). Theory of Edge Detection [J], Proceedings of the Royal Society London B207: 187-217.

Pursuing technology. (2006). Visual C++ digital image disposal typical arithmetic and implement $[\mathrm{M}]$. post \& telecom press.

Pavlidis, T. (1982). Algorithms for Graphics and Image Processing [J], Computer Science Press, Maryland, USA. 
T. Poggio, H. Voorhees and A. Yuille. (1985). A Regularized Solution to Edge Detection [J], May 1985 A. I. Memo 883, M.I.T.

Tou, J.T. \& R.C. Gonzalez.Pattern Recognition Principles [M], Addison-Wesley Publishing, Reading, MA, USA, 1981. 